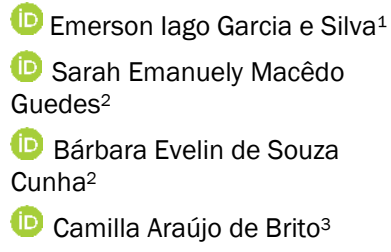

1 Universidade de Pernambuco, Programa de Pós-Graduação em Ciência e Tecnologia Ambiental para o Semiárido. Petrolina, PE, Brasil.

2 Faculdade Pernambucana de Saúde, Departamento de Nutrição. Recife, PE, Brasil.

${ }^{3}$ Instituto de Medicina Integral Professor Fernando Figueira, Departamento de Nutrição. Recife, PE, Brasil.

Correspondência

Emerson lago Garcia e Silva emerson.jago@hotmail.com

\section{Homeostase glicêmica e marcadores antropométricos associados à resistência insulínica em pacientes portadores de doença hepática gordurosa não alcoólica}

\author{
Glycemic homeostasis and anthropometric markers associated \\ with insulin resistance in patients with non-alcoholic fatty \\ liver disease
}

\begin{abstract}
Resumo
Objetivo: Determinar medidas antropométricas preditivas associadas à resistência à insulina em pacientes portadores de doença hepática gordurosa não alcoólica. Métodos: Estudo transversal, quantitativo, realizado em dois centros ambulatoriais de Recife-PE. O público foi formado por indivíduos de ambos os sexos, acima de 18 anos, com diagnóstico de doença hepática gordurosa não alcoólica via ecografia abdominal. As variáveis antropométricas coletadas foram: circunferência da cintura, índice de massa corporal, índice de conicidade, razão cintura-quadril e cintura-estatura. A resistência à insulina foi determinada através do Homeostasis model assessment: insulin resistance (HOMA-IR). Para entender as diferenças entre as variáveis, o teste de $U$ Mann-Whitney e testes de correlação foram realizados. Resultados: Participaram 75 indivíduos com predominância do sexo feminino (85\%) e com idade superior a 60 anos (44\%). A resistência à insulina foi elevada na população, perfazendo mais da metade dos indivíduos (73\%). Com exceção do índice de massa corporal e da razão cinturaquadril, demais índices apresentaram associação estatisticamente significativa entre a presença da resistência à insulina com a esteatose hepática não alcoólica: razão cintura-estatura ( $p=0,004)$, índice de conicidade $(p=0,031)$ e circunferência da cintura $(p=0,001)$. No teste de correlação, apenas a circunferência da cintura ( $r=0,2184$; $p=0,05)$ e a razão cintura-quadril ( $r=0,2310 ; p=0,04)$ associaram-se. Conclusões: Os indicadores antropométricos são ferramentas aplicáveis na prática clínica e no contexto de doença hepática gordurosa não alcoólica; contudo, a circunferência da cintura e as razões cintura-estatura e cintura-quadril apresentaram as melhores predições.
\end{abstract}

Palavras-chave: Antropometria. Doenças crônicas não transmissíveis. Resistência à insulina. Esteatose hepática.

Abstract
Objective: To determine predictive anthropometric measurements associated with
insulin resistance in patients with non-alcoholic fatty liver disease. Methods: This was a
cross-sectional, quantitative study conducted at two outpatient clinics in Recife-PE. The
study group was formed by individuals of both sexes over 18 years of age with non-
alcoholic fatty liver disease diagnosed via abdominal echography. The anthropometric
variables collected were: waist circumference, body mass index, conicity index, waist-
to-hip ratio, and waist-to-height ratio. Insulin resistance was determined through the
homeostasis model assessment: insulin resistance (HOMA-IR). The Mann-Whitney U 
test and correlation tests were performed to understand the differences between the variables. Results: 75 individuals participated in the study, most of whom were female (85\%) and with age over 60 years (44\%). Insulin resistance was high in the population, being present in more than half of the individuals (73\%). Except for the body mass index and waist-to-hip ratio, all other indices showed a significant association between the presence of insulin resistance and non-alcoholic hepatic steatosis: waist-to-height ratio $(p=0.004)$, conicity index $(p=0.031)$, and waist circumference $(p=0.001)$. In the correlation test, only the waist circumference $(r=0.2184 ; p=0.05)$ and the waist-to-hip ratio ( $r=0.2310 ; p=0.04$ ) were associated. Conclusions: The anthropometric indicators are applicable tools in clinical practice and in the context of non-alcoholic fatty liver disease; however, waist circumference and the waist-to-height and waist-to-hip ratios provided the best predictions.

Keywords: Anthropometry. Chronic non-communicable diseases. Insulin resistance. Hepatic steatosis. 


\section{INTRODUÇÃO}

A doença hepática gordurosa não alcoólica (DHGNA), uma condição clínica caracterizada pelo acúmulo anormal de gordura no fígado sem história de consumo significativo de álcool, é crescentemente reconhecida como a manifestação hepática da síndrome metabólica. ${ }^{2}$ É atualmente a causa mais comum de doença hepática nos países ocidentais e sua incidência vem aumentando nos países asiáticos. ${ }^{3,4}$ Compreende dois processos etiopatogênicos: a esteatose hepática não alcoólica (NAFL) e a esteato-hepatite não alcoólica (NASH). O NAFL é definido pela presença de esteatose hepática sem lesão hepatocelular na forma de hepatócitos em balão, enquanto a NASH é definida pela presença de esteatose hepática mais lesão e inflamação de hepatócitos. ${ }^{5}$

A DHGNA está intimamente relacionada às doenças metabólicas, como diabetes mellitus tipo 2 (DM2), resistência à insulina (RI) e obesidade, das quais a Rl é a base fisiopatológica comum. ${ }^{6}$ Além disso, achados anteriores mostraram que a DHGNA pode aumentar os riscos de DM2 e suas complicações em pacientes. ${ }^{7}$ De outro lado, eventos adicionais revelaram que as taxas de prevalência e mortalidade da DHGNA em pacientes com DM2 também foram significativamente maiores. ${ }^{8}$

A Rl é considerada um dos principais fatores de risco para doenças cardiovasculares, pois está associada a condições, como intolerância à glicose, DM2, dislipidemias, hipertensão arterial, entre outras alterações metabólicas, incluindo a DHGNA. ${ }^{9}$ Todavia, mesmo diante de sua importância para a saúde pública, a RI não é diagnosticada com frequência na prática clínica, ainda que importante para o diagnóstico, acompanhamento e tratamento de algumas doenças.

O método considerado padrão-ouro para a detecção dessa alteração é o clamp euglicêmico e hiperinsulinêmico, ${ }^{9}$ uma técnica de alto custo, invasiva e que demanda muito tempo, o que a inviabiliza para a prática clínica. Outro método utilizado é o Homeostasis model assessment: insulin resistance (HOMA-IR), que possui boa concordância com o teste padrão-ouro. No entanto, o HOMA-IR não é largamente empregado, pois valores de glicemia e insulinemia de jejum utilizados no seu cálculo demandam coletas invasivas, o que limita a praticidade do método. ${ }^{9}$

Diante disso, a necessidade de desenvolver métodos práticos, rápidos, não invasivos, de baixo custo e de fácil aplicação que possam se associar a resistência à insulina é crescente. Marcadores antropométricos obtidos empregando instrumentos simples e facilmente encontrados na prática profissional podem ser úteis nesse caso, pois alguns deles já possuem associação demonstrada com o acúmulo de gordura visceral. ${ }^{9}$

Sabendo da emergência enquanto problema de saúde pública da DHGNA e que um de seus gatilhos é a RI, são necessários estudos que avaliem os marcadores antropométricos relacionados, principalmente nesse público que tem crescimento alarmante e prognóstico desfavorável e insidioso. Desse modo, a identificação da RI merece maior atenção pelas entidades de saúde, uma vez que esta é uma forma de predizer importantes condições clínicas com enorme carga de morbi-mortalidade, tais como diabetes, eventos cardiovasculares e a própria DHGNA. Diante disso, a premissa deste trabalho foi determinar medidas antropométricas preditivas associadas à resistência à insulina em pacientes portadores de doença hepática gordurosa não alcoólica.

\section{MÉTODO}

\section{Aspectos éticos}

Esta pesquisa constitui um recorte de um projeto maior intitulado "Parâmetros clínicos e nutricionais de pacientes portadores de esteatose hepática não alcoólica e sua relação com a síndrome metabólica", aprovado pelo Comitê de Ética em Pesquisa do Instituto de Medicina Integral Professor Fernando Figueira, sob o número: 
64898517.1.0000.5201. Todos os participantes foram esclarecidos sobre a natureza e perspectivas do projeto. A participação dos indivíduos ocorreu mediante assinatura do Termo de Consentimento Livre e Esclarecido (TCLE).

\section{Delineamento do estudo}

Este é um estudo de natureza transversal, multicêntrico, com abordagem quantitativa, sendo realizado nos ambulatórios de Nutrição do Instituto de Medicina Integral Professor Fernando Figueira e Instituto do Fígado de Pernambuco, ambos, centros de atendimento à população local e áreas afins, localizados em Recife-PE, Brasil.

A coleta de dados teve início em abril e término em novembro de 2017. A amostragem foi não probabilística por conveniência, com pacientes atendidos nas unidades. Como critérios de inclusão, fizeram parte do cômputo amostral apenas indivíduos com diagnóstico de esteatose hepática não alcoólica via ecografia abdominal (USG), de ambos os sexos e acima de 18 anos. Foram excluídos: etilistas ativos, sujeitos em uso de medicação esteatogênica ou hepatotóxica, com diagnóstico de alguma desordem hepática hereditária ou soropositividade para vírus da hepatite B (HVB) e C (HVC), e pacientes com alguma doença incapacitante (reumáticas, neuromusculares, osteoarticulares ou degenerativas). Toda informação foi checada em prontuário médico, para verificar a veracidade conforme anamnese realizada nos participantes.

\section{Medidas e índices antropométricos}

As medidas antropométricas realizadas no primeiro encontro foram: peso (Kg), altura (m), circunferência da cintura (CC) e circunferência do quadril (CQ). Ambas circunferências foram estabelecidas em centímetros (cm). O peso foi mensurado em balança digital (marca Welmy®, modelo W300) com estadiômetro acoplado, capacidade para $300 \mathrm{~kg}$ e precisão de 50g, com o indivíduo portando roupas leves, posicionado de costas, no centro do equipamento, ereto, com os pés juntos e os braços estendidos ao longo do corpo, com o olhar para frente, mantendo-se parado nessa posição.

A altura ( $\mathrm{m}$ ) foi realizada em posição ortogonal, com o indivíduo ereto e de costas para o equipamento, a parte móvel do estadiômetro posicionada fixando-a contra a cabeça, com pressão suficiente para comprimir o cabelo. A CC foi realizada com o paciente em pé utilizando uma fita métrica inelástica ao nível do ponto médio entre a última costela e a crista ilíaca. ${ }^{10}$ Esses valores foram analisados conforme os critérios da Organização Mundial da Saúde (OMS). ${ }^{11}$ A circunferência do quadril foi aferida na máxima extensão glútea que pudesse ser vista. ${ }^{10}$

A partir das medidas antropométricas, foram calculados os seguintes índices:

1) Índice de massa corporal (IMC) - calculado pela razão entre o peso corporal (kg) e altura ao quadrado ( $\left.\mathrm{m}^{2}\right)$. A interpretação se baseou nos critérios da OMS ${ }^{12}$ para não idosos. A população foi estratificada considerando IMC em: baixo peso (<18.5 kg/m²); peso normal ou eutrofia $\left(18.5 \mathrm{~kg} / \mathrm{m}^{2}-24.9 \mathrm{~kg} / \mathrm{m}^{2}\right)$; sobrepeso $\left(25 \mathrm{~kg} / \mathrm{m}^{2}-29,9 \mathrm{~kg} / \mathrm{m}^{2}\right)$; obesidade $\left(\geq 30 \mathrm{~kg} / \mathrm{m}^{2}\right)$. A categorização dos idosos seguiu o que propõe a Organização Pan-Americana da Saúde (OPAS): ${ }^{13}$ baixo peso (IMC $\left.<23 \mathrm{~kg} / \mathrm{m} 2\right)$; peso adequado (IMC $\geq 23$ e $<28 \mathrm{~kg} / \mathrm{m}^{2)}$; pré-obesidade $\left(\mathrm{IMC}>28\right.$ e $<30 \mathrm{~kg} / \mathrm{m}^{2)}$; obesidade (IMC $\geq 30 \mathrm{~kg} / \mathrm{m} 2)$.

2) Índice de conicidade (IC) - calculado através das medidas peso, estatura, circunferência da cintura e uma constante que resulta da raiz da razão entre $4 \pi$ (originado da dedução do perímetro do círculo de um cilindro) e a densidade média do ser humano de $1.050 \mathrm{~kg} / \mathrm{m}^{3}$. A descrição acima tem a seguinte fórmula esquematizada: IC: CC/0.109XVPeso (Kg)/Estatura (cm). Com os pontos de corte de 1,25 para homens, 1,18 e 1,22 para mulheres até 49 anos e a partir de 50 anos de idade, respectivamente. ${ }^{14}$ 
3) Razão cintura-quadril (RCQ), calculada dividindo-se o valor da medida da circunferência da cintura (CC) pelo resultado da circunferência do quadril, e que atribui risco metabólico e cardiovascular valores $\geq 0,90$ para homens e $\geq 0,85$ para mulheres.

4) $O$ índice razão cintura-estatura (RCE), matematicamente obtido pelo quociente entre a circunferência da cintura (cm) e a estatura (cm). Devido à inexistência, até o presente momento, de uma referência nacional de pontos de corte do RCE, considerou-se o percentil (P) 90, que corresponde a 0,50, como limite para o diagnóstico do excesso de gordura abdominal para adultos. No que tange aos idosos, utilizou-se o ponto de corte de 0,55 proposto por Correa. ${ }^{15}$

\section{Avaliação bioquímica e resistência à insulina}

Foram coletadas dosagens séricas de glicemia em jejum e insulina plasmática, obtidas através dos prontuários médicos, considerando um período máximo de três meses. Na ausência desses exames, eram realizadas solicitações para coleta e análise, ocorrendo na primeira consulta.

Em relação à glicemia em jejum, os critérios da Sociedade Brasileira de Diabetes foram adotados a fim de estratificar a normalidade ou inflexão. ${ }^{16}$ Os valores de insulina foram considerados normais no intervalo de 3 a 25 um/L, conforme especificações do laboratório. A resistência à insulina foi determinada pelo Homeostasis model assessment: insulin resistance (HOMA-IR), obtida pela fórmula17: HOMA = [Insulinemia de jejum (mU/l) $\times$ Glicemia de jejum (mmol/l)] / 22,5. Para definir resistência à insulina, considerou-se um valor maior que 2.7, de acordo com um estudo de prevalência realizado com a população brasileira. ${ }^{18}$

\section{Análise estatística}

Os dados foram compilados no Microsoft Excel 2013 para o delineamento estatístico descritivo e uso nos programas de inferência. Na análise inferencial, as variáveis contínuas foram testadas quanto à distribuição normal pelo teste de normalidade de Kolmogorov-smirnov. A homocedasticidade não foi atingida, sendo aplicado o teste de U Mann-Whitney para testar as diferenças entre as medianas dos grupos independentes. Para analisar a correlação das variáveis, o teste de Spearman foi utilizado para aquelas com distribuição não normal, e o teste de Pearson para as variáveis que apresentaram distribuição normal. Todas as análises foram realizadas usando o programa SPSS versão 23.0(SPSS Inc., Chicago, IL, USA). Valores de P bicaudais <0,05 foram considerados estatisticamente significativos.

\section{RESULTADOS}

Foram pré-selecionados 141 participantes com diagnóstico de esteatose hepática não alcoólica via USG, dos quais um positivou para HVB, um não permitiu completa mensuração antropométrica e 64 indivíduos não apresentaram alguns exames bioquímicos solicitados, o que resultou numa amostragem de 75 indivíduos adultos de ambos os sexos elegíveis. Destes, prevaleceu o sexo feminino (85\%). Com relação à idade, $44 \%$ dos indivíduos apresentavam mais que 60 anos, mas o público de meia-idade, entre 45 e 59 anos, respondeu por $40 \%$ da amostra. Apenas 16\% eram considerados adultos jovem, ou seja, com menos de 45 anos.

Em relação ao grau de esteatose na amostra, 36 (48\%) indivíduos apresentavam acúmulo leve, seguido por moderado (43\%) e severo (9\%). 
Tabela 1. Perfil antropométrico e bioquímico de pacientes com doença hepática gordurosa não alcoólica. RecifePE, 2017.

\begin{tabular}{|c|c|c|c|}
\hline Variável & $\mathrm{N}$ & $\%$ & Min-Máx. \\
\hline \multicolumn{4}{|l|}{$I M C\left(\mathrm{~kg} / \mathrm{m}^{2}\right)$} \\
\hline Eutrofia & 8 & 11 & $24,5-27,3$ \\
\hline Sobrepeso & 14 & 19 & $24,9-29,9$ \\
\hline Obesidade & 53 & 70 & $30,5-42,5$ \\
\hline \multicolumn{4}{|l|}{$R C E(\mathrm{~cm})$} \\
\hline Normal & 0 & 0 & - \\
\hline Elevada & 75 & 100 & $0,53-0,92$ \\
\hline \multicolumn{4}{|l|}{$R C Q(\mathrm{~cm})$} \\
\hline Normal & 6 & 8 & $0,74-0,93$ \\
\hline Elevada & 69 & 92 & $0,83-1,29$ \\
\hline \multicolumn{4}{|l|}{ IC } \\
\hline Normal & 14 & 19 & $1,04-1,62$ \\
\hline Alterado & 61 & 81 & $1,00-1,79$ \\
\hline \multicolumn{4}{|l|}{$C C(\mathrm{~cm})$} \\
\hline Normal & 0 & 0 & - \\
\hline Elevado & 10 & 13 & $86,0-99,0$ \\
\hline Muito Elevado & 65 & 87 & $88,0-140$ \\
\hline \multicolumn{4}{|c|}{ Glicemia em Jejum (mg/dL) } \\
\hline Normal & 45 & 60 & 74-99 \\
\hline Alterado & 30 & 40 & $103-364$ \\
\hline \multicolumn{4}{|c|}{ Insulinemia em Jejum (mU/L) } \\
\hline Normal & 62 & 83 & $3,00-24,3$ \\
\hline Alterado & 13 & 17 & $25,02-221,1$ \\
\hline \multicolumn{4}{|c|}{ Resistência à insulina (HOMA - IR) } \\
\hline Não & 20 & 27 & $0,6-2,5$ \\
\hline Sim & 55 & 73 & $2,8-52,2$ \\
\hline
\end{tabular}

Legenda: IMC: Índice de massa corporal; RCE: Razão cintura-estatura; RCQ: Razão cintura-quadril; IC: Índice de conicidade; CC: Circunferência da cintura; HOMA - IR: Homeostasis model assessment: insulin resistance; Min-Máx: Mínimo-Máximo.

Na tabela 1, sumarizam-se as características antropométricas e bioquímicas dos indivíduos portadores de DHGNA. A obesidade foi prevalente na amostra, como apontado pelas medidas antropométricas avaliadas, sendo o excesso de gordura abdominal encontrado em todos os indivíduos estudados, dos quais $80 \%$ apresentavam CC muito elevada. A resistência à insulina segundo HOMA-IR foi elevada na população, perfazendo mais da metade dos indivíduos (73\%). 
Tabela 2. Associação da resistência à insulina com variáveis antropométricas e bioquímicas em indivíduos portadores de doença hepática gordurosa não alcoólica. Recife-PE, 2017.

\begin{tabular}{|c|c|c|c|}
\hline \multirow[b]{2}{*}{ Variável } & \multicolumn{2}{|c|}{ Resistência à insulina } & \multirow[b]{2}{*}{$p$-valor ${ }^{*}$} \\
\hline & $\begin{array}{l}\text { Presença } \\
(\mathrm{N}=55)\end{array}$ & $\begin{array}{c}\begin{array}{c}\text { Ausência } \\
(\mathrm{N}=20)\end{array} \\
\end{array}$ & \\
\hline & Mediana (IQ) & Mediana (IQ) & \\
\hline $\mathrm{IMC}\left(\mathrm{kg} / \mathrm{m}^{2}\right)$ & $32,6(29,5-37)$ & $32,15(29,5-34)$ & 0,429 \\
\hline RCE (cm) & $0,67(0,0-0,0)$ & $0,65(0,0-0,0)$ & $0,004^{*}$ \\
\hline $\mathrm{RCQ}(\mathrm{cm})$ & $0,97(0,0-1,0)$ & $0,93(0,0-0,25)$ & 0,143 \\
\hline IC & $1,34(1,0-1,0)$ & $1,29(1,0-1,0)$ & 0,031 * \\
\hline CC (cm) & $103,5(99,5-115)$ & $102(87,7-103)$ & 0,001 * \\
\hline HOMA - IR & $4,6(3,0-6,5)$ & $1,7(1,0-2,0)$ & $<0,000^{*}$ \\
\hline
\end{tabular}

Legenda: IMC: Índice de massa corporal; RCE: Razão cintura-estatura; RCQ: Razão cintura-quadril; IC: Índice de conicidade; CC: Circunferência da cintura; IQ: Intervalo interquartil; *:Valores estatisticamente significante pelo teste U Mann-Whitney.

A associação da resistência à insulina com os parâmetros antropométricos pode ser observada na tabela 2. Dos indicadores antropométricos, RCE $(p=0,0048)$, IC ( $p=0,0310)$ e CC $(p=0,0011)$ foram estatisticamente significantes, sendo as medianas superiores no grupo com Rl em relação ao grupo sem a manifestação. Em relação aos níveis de glicemia $(<0,0001)$ e insulina em jejum $(<0,0001)$, a tendência aumentada foi mantida e com significância estatística nos grupos com RI. O mesmo foi observado para o HOMA-IR $(<0,0001)$, significativamente maior no grupo com a condição clínica de RI.

Tabela 3. Correlação entre indicadores antropométricos e bioquímicos com resistência á insulina segundo HOMA-IR em indivíduos portadores de doença hepática gordurosa não alcoólica. Recife, Pernambuco, 2017.

\begin{tabular}{ll}
\hline Indicadores & $r$ \\
\hline IMC $\left(\mathrm{kg} / \mathrm{m}^{2}\right)$ & 0.1458 \\
RCE $(\mathrm{cm})$ & 0.1310 \\
RCQ (cm) & $0.2310^{*}$ \\
IC & 0.0631 \\
CC (cm) & $0.2184^{*}$ \\
Glicemia em jejum & 0.1785
\end{tabular}

Legenda: IMC: Índice de Massa Corporal; RCE: Razão cintura-estatura; RCQ: Razão cintura-quadril; IC: Índice de conicidade; CC: Circunferência da cintura. *:Diferenças estatisticamente significante pelo teste de Correlação de Spearman 
A tabela 3 resume a força de associação através da correlação entre a resistência à insulina e os marcadores antropométricos e bioquímicos. Correlações positivas significativas com a RI foram alcançadas apenas para CC $(p=0,05)$ e RCQ $(p=0,04)$. Ressalta-se que, para as medidas antropométricas, o valor de R não foi tão elevado, evidenciando que a relação não foi forte. Reitera-se que a população do estudo pode ter sido pequena para testar tal efeito e uma amostragem maior possibilitaria melhor evidência.

\section{DISCUSSÃO}

A DHGNA é uma das principais condições de saúde em todo o mundo, afinal 30\% da população adulta e 60\% a $80 \%$ dos pacientes diabéticos e obesos são afetados. ${ }^{19,20}$ Trata-se de uma doença progressiva crônica e sua evolução geralmente leva anos ou até décadas. ${ }^{21}$ Ainda assim, as evidências sugerem que a DHGNA é a principal causa de carcinoma hepatocelular nos Estados Unidos. ${ }^{22}$ Somado a isso, sua importância como segundo evento principal causador de transplante hepático é uma realidade..$^{23}$ Além de transtornos de ordem hepática, é também responsável pelo incremento de casos de DM2, síndrome metabólica e eventos cardiovasculares. ${ }^{21}$

A RI tem sido reconhecida como fundamental no desenvolvimento da esteatose hepática não alcoólica. Nesse sentido, sabe-se que o desequilíbrio resultante da resistência à insulina no metabolismo de lipídios acarreta deposição de gordura no fígado, devido a redução da oxidação de ácidos graxos livres e/ou aumentada lipogênese hepática de novo e/ou redução da liberação de lipídios para a circulação. Consequentemente, os depósitos de gordura no hepatócito aumentam a resistência à insulina, formando um círculo vicioso. E ainda, a hiperinsulinemia é resultante da predisposição genética, do excesso de oferta de ácidos graxos livres ou da exposição a níveis elevados de fator de necrose tumoral-alfa (TNF-a), interleucina 6 (IL-6) e/ou outros mediadores. ${ }^{24}$

Nesta pesquisa, a RI foi numericamente importante, assumindo percentuais elevadas (73,4\%). Estudo brasileiro realizado no Rio de Janeiro encontrou valores de 65\% dessa manifestação e uma média de 4.4 no HOMAIR, em indivíduos obesos com DHGNA. ${ }^{25}$ Em nosso estudo, encontramos valores de HOMA-IR superiores; entretanto, em ambos os estudos, o ponto de corte estabelecido para RI foi superado. Outro estudo nacional, realizado em Porto Alegre, observou valores médios elevados de HOMA-IR de 5.5, corroborando nossos achados. ${ }^{26}$ Já o estudo de Barros e colaboradores, que buscou relacionar a DHGNA com síndrome metabólica em obesos mórbidos, encontrou 86.8\% de HOMA-IR anormal. ${ }^{27}$ Entretanto, o referido estudo trabalhou apenas com obesos mórbidos, que conhecidamente apresentam pior homeostase glicêmica, fator que pode ter contribuído para um número tão elevado e diferente de nossa amostra, de característica mais heterogênea quanto ao estado nutricional.

De modo geral, a persistência e relação da RI e DHGNA é também observada no cenário internacional. As Divisões de Hepatologia e Gastroenterologia do Texas e da Flórida, EUA, buscando reconhecer o papel determinante da DHGNA e RI e da esteato-hepatite na dislipidemia aterogênica, encontraram valores de 4.9 no HOMA-IR. ${ }^{28}$ Tal

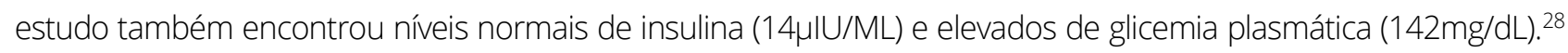
Em nosso estudo, 40\% dos indivíduos apresentaram glicemia alterada, enquanto a média dos grupos com RI foi $122 \mathrm{mg} / \mathrm{dL}$.

Tal analogia entre valores elevados reforça a RI com hiperglicemia como enlace, assemelhando nossa pesquisa ao estudo internacional supracitado. Por outro lado, no estudo citado, os valores de insulina média permaneceram dentro da faixa de normalidade. ${ }^{26}$ Em nossa pesquisa o mesmo foi observado, uma vez que grande parte da nossa população permaneceu na faixa de normalidade insulinêmica. Quanto aos valores médios, em ambos os grupos, com e sem Rl, a insulinemia basal encontrava-se dentro da normalidade, considerando a mediana populacional, embora significativamente superior no grupo com RI. Esse comportamento de "normalidade insulínica" majoritário foi constatado em outros ensaios. ${ }^{25,27}$ Tais resultados reforçam a criticidade e cautela em considerar os níveis de insulina num contexto de DHGNA e RI como forma diagnóstica e/ou monitoramento clínico exclusivo. 
As medidas antropométricas são indicadores do estado nutricional, apresentam baixo custo, inocuidade, simplicidade em sua execução e têm servido como indicadores da obesidade. As correlações entre indicadores antropométricos e RI têm sido vastamente estudadas, destacando-os como indicadores não invasivos para a avaliação do risco de Rl, tanto na pesquisa epidemiológica como na prática clínica. ${ }^{9}$

A depender da medida antropométrica utilizada, seus resultados podem relatar diferentes gravidades de acúmulo de gordura no corpo, sendo importante distinguir o grau de gordura generalizada e gordura abdominal. Como marcadores de obesidade central ou gordura abdominal, podemos citar CC, IC, RCE e RCQ. Já entre os marcadores de obesidade generalizada, destaca-se o IMC.

A obesidade centralizada é um fator de risco cardiovascular importante, além de ter maior interferência na homeostase glicose-insulina do que a obesidade generalizada. ${ }^{29}$ Analisando os achados deste estudo, percebe-se que dentre os marcadores de obesidade centralizada, RCE e IC associaram-se significativamente com a presença de insulina na amostra com DHGNA, enquanto a CC mostrou tendência estatística. Por outro lado, o IMC, que indica generalização de gordura, e a RCQ não mostraram associação. De outro lado, a RCQ $(r=0,2310 ; p=0,04)$ e a CC $(r=0,2184 ; p=0,05)$ apresentaram uma correlação positiva significativa com os valores do HOMA-IR.

Estudo brasileiro realizado em Minas Gerais, procurando identificar os marcadores antropométricos de RI em homens idosos, encontrou correlações positivas de todos os índices antropométricos com o HOMA-IR. ${ }^{30}$ São resultados divergentes do nosso trabalho, mas é importante mencionar que nesse estudo brasileiro a população não apresentava diagnóstico de DHGNA, o que revela que para esse público alguns parâmetros antropométricos apresentam melhor associação que outros, o que não é visto em pacientes sem a DHGNA preexistente.

A circunferência da cintura aumentada se associou com a presença Rl e correlacionou-se com os valores do HOMA-IR. Um estudo iraniano também confirmou a relação da RI com a CC, sendo esta mais elevada naqueles com a manifestação. ${ }^{31}$ Tal resultado corrobora os achados deste estudo em relação a associação e correlação da RI com CC.

Ainda no cenário internacional, estudo transversal realizado com homens japoneses com faixa etária de 50 anos em média observou também associação da CC com RI. ${ }^{32}$ Semelhantemente, estudo prospectivo mexicano também encontrou forte associação da CC com RI e DM2. ${ }^{33}$ De modo geral, os estudos convergem, incluindo este, para anormalidades elevadas da CC e respostas glicêmicas anormais.

O IC e a RCE têm-se mostrado importantes ferramentas de indicação de risco cardiovascular. Por outro lado, sua utilização na identificação de Rl é ainda muito incipiente. Em nosso estudo, observamos que ambos os índices se associaram com a presença de Rl, apresentando-se significativamente aumentados naqueles com a manifestação. ${ }^{34,35}$

Estudo multicêntrico realizado com sete populações europeias e duas americanas comparou o IC e RCE como indicadores de saúde cardiovascular. Em tal estudo, verificou-se que a insulinemia apresentou padrões de correlação consistentes com o IC.34 Semelhança foi observada em outro estudo nacional, no qual os autores identificaram o IC como discriminador de glicemia e de risco cardiovascular. ${ }^{13}$ Tais achados estão em desacordo com o presente estudo. Em contrapartida, em um estudo realizado na Grécia com 280 mulheres saudáveis de 18 a 24 anos, o IC apresentou correlação muito fraca ( $r=0,13 ; p=0,03)$ com a insulinemia de jejum. ${ }^{35}$ As evidências são inconclusivas quanto ao uso do IC na RI em pacientes com DHGNA. Existem poucos estudos nesse arco temático, dificultando qualquer sugestão. A priori, e com base em nossos achados, o IC é significativamente maior em indivíduos com Rl.

Um trabalho brasileiro com pacientes diagnosticados com síndrome coronariana aguda encontrou uma correlação negativa da RCE com a RI. ${ }^{36}$ Esse achado entra duplamente em desacordo com nosso trabalho, primeiro 
porque não foi encontrado uma correlação significativa, e segundo, o tipo de correlação foi positiva. Embora a RCE seja considerada superior ao IMC e à CC, por ser sensibilizada pelo sexo e raça pode inflexionar os resultados. Isso porque a distribuição de gordura varia conforme sexo e raça, o que minimiza a capacidade da RCE em discriminar a Rl. Como são escassos os estudos que utilizam a RCE com a finalidade de identificar a Rl, surge a dificuldade de refutar ou não a hipótese.

Alguns indicadores antropométricos tradicionais podem não estratificar corretamente o risco metabólico associado à obesidade, inclusive em pacientes obesos. ${ }^{37}$ Isso foi observado com o IMC, que não se associou com a Rl e tampouco se correlacionou neste estudo, uma vez que sua maior limitação reside em correlacionar-se com a gordura corporal total, não refletindo a distribuição da gordura corporal. ${ }^{38}$ Além disso, existe uma tendência do IMC em subestimar a adiposidade em idosos e superestimar em indivíduos com maior quantidade de massa magra, inviabilizando ainda mais seu poder discriminante. ${ }^{39}$

Pelo exposto acima, este estudo teve como ponto forte abordar parâmetros da prática nutricional em um público com DHGNA cuja prevalência é crescente. Além disso, revela que alguns são mais preditivos que outros em associar-se à Rl, um dos fatores no desenvolvimento da doença. Como limitações, cabe ressaltar que a pequena amostra pode ter sido insuficiente para testar com maior evidência as associações.

\section{CONCLUSÃO}

Os indicadores antropométricos são ferramentas aplicáveis na pratica clínica e no contexto da DHGNA. O excesso de gordura abdominal se relaciona com a RI como foi observado pelas associações da RCE, IC e CC, elevados significativamente no grupo com Rl. Além disso, a gordura central exerce papel importante.

Os achados mostram uma ligação diretamente proporcional da CC e RCQ com o HOMA-IR. Sugere-se que os marcadores antropométricos, especificamente aqueles que delimitam a distribuição de gordura central, como CC, RCQ e RCE, tenham papel relevante no acompanhamento da Rl em indivíduos com DHGNA.

\section{AGRADECIMENTOS}

À Secretaria Estadual de Saúde de Pernambuco, pelo apoio financeiro.

\section{REFERÊNCIAS}

1. Wijarnpreecha $K$, Panjawatanan $P$, Thongprayoon $C$, Jaruvongvanich $V$, Ungprasert $P$. Sarcopenia and risk of nonalcoholic fatty liver disease: A meta-analysis. Jornal Oficial da Saudi Gastroenterology Association. 2018;24(1):12-7. https://doi.org/10.4103/sjg.SJG_237_17

2. Younossi ZM, Koenig AB, Abdelatif D, Fazel Y, Henry L3, Wymer M. Global epidemiology of nonalcoholic fatty liver disease-Meta-analytic assessment of prevalence, incidence, and outcomes. Hepatology. 2016;64:73-84.

3. Seki Y, Kakizaki S, Horiguchi N, Hashizume H, Tojima H, Yamazaki Y, Sato K, Kusano M, Yamada M, Kasama K. Prevalence of nonalcoholic steatohepatitis in Japanese patients with morbid obesity undergoing bariatric surgery. J Gastroenterol. 2016;51(3):281-9. https://doi.org/10.1007/s00535-015-1114-8

4. Watanabe S1, Hashimoto E, Ikejima K, Uto H, Ono M, Sumida Y, Seike M, Takei Y, Takehara T, Tokushige K, Nakajima A, Yoneda M, Saibara T, Shiota G, Sakaida I, Nakamuta M, Mizuta T, Tsubouchi H, Sugano K, Shimosegawa T. Evidence-based clinical practice guidelines for nonalcoholic fatty liver disease/nonalcoholic steatohepatitis. J Gastroenterol. 2015;50(4):364-77.https://doi.org/ 10.1007/s00535-015-1050-7

5. Tokushige K, Hashimoto E, Kodama K. Hepatocarcinogenesis in non-alcoholic fatty liver disease in Japan. J Gastroenterol Hepatol. 2013;28(4):88-92. https://doi.org: 10.1111/jgh.12239

6. Fruci B, Giuliano S, Mazza A, Malaguarnera R, Belfiore A.Nonalcoholic Fatty liver: a possible new target for type 2 diabetes prevention and treatment. Int J Mol Sci. 2013;14(11):22933-66. https://doi.org /10.3390/ijms141122933 
7. Mavrogiannaki AN, Migdalis IN. Nonalcoholic Fatty liver disease, diabetes mellitus and cardiovascular disease: newer data. Int J Endocrino. 2013;2013(450639):1-8. http://dx.doi.org/10.1155/2013/450639

8. Targher G, Bertolini L, Poli F, Rodella S, Scala L, Tessari R, Zenari L, Falezza G. Nonalcoholic fatty liver disease and risk of future cardiovascular events among type 2 diabetic patients. Diabetes. 2005;54(12):3541-46.

9. Carneiro IBP, Sampaio HAC, Carioca AAF, Pinto FJM, Damasceno NRT.Antigos e novos indicadores antropométricos como preditores de resistência à insulina em adolescentes. Arq Bras Endocrinol Metab. 2014;58(8):838-43. http://dx.doi.org/10.1590/0004-2730000003296

10. Mussoi TD. Avaliação Nutricional na Prática Clínica: da gestação ao envelhecimento. Rio de Janeiro: Guanabara; 2014. p. 423.

11. World Health Organization. Obesity: preventing and managing the global epidemic. WHO; 1998.

12. World Health Organization. Obesity: preventing and managing the global epidemic. Report of a World Health Organization Consultation. Geneva: World Health Organization; 2000. p. 256. WHO Obesity Technical Report Series, ก. 284.

13. OPAS. Organização Pan-Americana. XXXVI Reunión del Comitê Asesor de Ivestigaciones en Salud - Encuestra Multicêntrica - Salud Beinestar y Envejecimeiento (SABE) en América Latina e el Caribe - Informe preliminar; 2002

14. Pitanga FJG, Lessa I. Sensibilidade e especificidade do índice de conicidade como discriminador do risco coronariano de adultos em Salvador, Brasil. Rev Bras Epidemiol. 2004;7(3):259-69. http://dx.doi.org/10.1590/S1415$790 \times 2004000300004$

15. Correa MM, Thomasi E, Tume E, Oliveira ERA, Facchini LA. Razão cintura-estatura como marcador antropométrico de excesso de peso em idosos brasileiros. Cad. Saúde Pública. 2017;33(5):1-14. http://dx.doi.org/10.1590/0102$311 \times 00195315$.

16. Diretrizes Sociedade Brasileira de Diabetes (2019-2020). Rio de Janeiro: Clannad; 2019. p.491.

17. Matthews DR, Hosker JP, Rudenski AS, Naylor BA, Treacher DF, Turner RC. Homeostasis model assessment: insulin resistance and beta-cell function from fasting plasma glucose and insulin concentrations in man. Diabetologia. 1985;28(7):412-19

18. Geloneze B, Vasques AC, Stabe CF, Pareja JC, Rosado LE, Queiroz EC, Tambascia MA. HOMA1-IR and HOMA2-IR indexes in identifying insulin resistance and metabolic syndrome: Brazilian Metabolic Syndrome Study (BRAMS). Arquivos Brasileiros de Endocrinologia \& Metabologia. 2009;53(2):281-87.

19. Ratziu V, Bellentani S, Cortez-Pinto H, Day C, Marchesini G. A position statement on NAFLD/NASH based on the EASL 2009 special conference. Journal of Hepatology. 2010;53(2):72-384. https://doi.org: 10.1016/j.jhep.2010.04.008

20. Younossi ZM, Stepanova M, Afendy M, Fang Y, Younossi Y, Mir H, Srishord M. Mudanças na prevalência das causas mais comuns de doenças crônicas do fígado nos Estados Unidos de 1988 a 2008. Clinical Gastroenterology and Hepatology. 2011;9(6):524-30. https:doi.org: 10.1016/j.cgh.2011.03.020

21. Dai $H$, Wang $W$, Chen $R$, Chen Z, Lu Y, Yuan H. Lipid accumulation product is a powerful tool to predict non-alcoholic fatty liver disease in Chinese adults. Nutrition \& Metabolism.2018;14(49):1-9. https://doi.org: 10.1186/s12986-0170206-2

22. Yu J, Shen J, Sun $T$, Zhang $X$, Wong $N$. Obesity, insulin resistance, NASH and hepatocellula carcinoma. Semin Câncer Biol. 2013;23(6):483-91.https:doi.org:10.1016/j.semcancer.2013.07.003

23. Wong RJ, Aguilar M, Cheung R, Perumpail RB, Harrison SA, Younossi ZM, Ahmed A. Nonalcoholic steatohepatitis is the second leading etiology of liver disease among adults awaiting liver transplantation in the United States. Gastroenterology. 2015;148(3):547-55. https:doi.org: 10.1053/j.gastro.2014.11.039

24. Portela CLM, Melo MLP, Sampaio HAC. Aspectos fisiopatológicos e nutricionais da doença hepática gordurosa nãoalcoólica (DHGNA). Rev Bras Nutr Clin. 2013;28(1):54-60.

25. Chaves VG, Souza DS, Pereira SE, Saboya CJ, Peres WAF. Associação entre doença hepática gordurosa não alcoólica e marcadores de lesão/função hepática com componentes da síndrome metabólica em indivíduos obesos classe III. Rev Assoc Med Bras. 2012;58(3):288-293. http://dx.doi.org/10.1590/S0104-42302012000300007

26. Leon LB, Becker SCC, Petry RC, Wink EF, Lantz F, Tovo CV. Doença hepática gordurosa não alcoólica em pacientes com índice de massa corporal normal: etiologia e fatores de risco associados em um hospital terciário. Revista da AMRIGS. 2014;58(1):44-48 
27. De Barros F, Setúbal S, Martinho JM, Ferraz L, Gaudêncio A. Barros, F et al. Correlation of non-alcoholic fatty liver disease and features of metabolic syndrome in morbidly obese patients in the preoperative assessment for bariatric surgery. ABCD Arq Bras Cir Dig. 2016;29(4):260-63. https://doi.org:10.1590/0102-6720201600040011.

28. Bril F, Sninsky J, Baca AM, Superko HR, Portillo Sanchez P, Biernacki D, Maximos M, Lomonaco R, Orsak B, Suman A, Weber MH, McPhaul MJ, Cusi K. Hepatic Steatosis and Insulin Resistance, But Not Steatohepatitis, Promote Atherogenic Dyslipidemia in NAFLD. J Clin Endocrinol Metab. 2016;101(2):644 - 52. https:doi.org:10.1210/jc.20153111

29. Martins IS, Marinho SP. O potencial diagnóstico dos indicadores da obesidade centralizada. Rev Saúde Pública. 2003;37(6):760-767. http://dx.doi.org/10.1590/S0034-89102003000600011

30. Anunciação PM, Ribeiro RCL. Anthropometric indicators in identification of insulin resistance in elderly men. Mundo da Saúde. 2015;39(2):157-163.http://doi.org/10.15343/0104-7809.20153902157163

31. Zadeh-Vakili A, Teerã FR, Hosseinpanah F. Waist circumference and insulin resistance: a community based cross sectional study on reproductive aged Iranian women. Diabetologia e Síndrome Metabólica. 2011;3(18):2-6. https://doi.org/10.1186/1758-5996-3-18

32. Tabata S, Yoshimitsu S, Hamachi T, Abe H, Ohnaka K, Kono S. Waist circumference and insulin resistance: a crosssectional study of Japanese men. BMC Endocrine Disorders. 2009;9(1):1-7. https://doi.org:10.1186/1472-6823-9-1.

33. Mamtani M, Kulkarni H, Dyer TD, Almasy L, Mahaney MC, Duggirala R, Comuzzie AG, Blangero J, Curran JE. Waist Circumference Independently Associates with the Risk of Insulin Resistance and Type 2 Diabetes in Mexican American Families. PLOS ONE. 2013;8(3):1-7. https://doi.org:10.1371/journal.pone.0059153.

34. Valdez R, Seidell JC, Ahn YI, Weiss KM. A new index of abdominal adiposity as na indicator of risk for cardiovascular disease. A cross-population study. IntJObesRel Met Disorders. 1993;17(2):77- 82.

35. Mantzoros CS, Evagelopoulou K, Georgiadis El, Katsilambros N. Conicity index as a predictor of blood pressure levels, insulin and triglyceride concentrations of healthy premenopausal women. Norm Metab Res. 1996;28(1):32-4.

36. Marcadenti A, Oliveira VG, Bertoni VM, Wittke E, Dourado LP, Souza RB, Pinto TM, Filho PP, Leivas JASL. Resistência à Insulina e Indicadores Antropométricos em Pacientes com Síndrome Coronariana Aguda. Rev Bras Cardiol. 2013;26(4):259-66

37. Lemieux I, Drapeau V, Richard D, PHD13, Bergeron J, Marceau P, Biron S, Mauriège P. Waist girth does not predict metabolic complications in severely obese men. Diabetes Care. 2006;29(6):1417-9. https://doi.org/10.2337/dc060441

38. Son YJ, Kim J, Park HJ, Park SE, Park CY, Lee WY, Oh KW, Park SW, Rhee EJ. Association of Waist-Height Ratio with Diabetes Risk: A 4-Year Longitudinal Retrospective Study. Endocrinology and Metabolism. 2016;31(1):127-133. https://doi: 10.3803/EnM.2016.31.1.127

39. Antonini-Canterin F, Di Nora C, Poli S, Sparacino L, Cosei L, Ravasel A, AC Popescu, Popescu BA. Obesity, Cardiac Remodeling, and Metabolic Profile: Validation of a New Simple Index beyond Body Mass Index. Journal of Cardiovascular Echography.2018;28(1):18-25. https://doi.org:10.4103/jcecho.jcecho_63_17

\section{Colaboradores}

Silva EIG foi responsável pela concepção, desenho, coleta, análise e interpretação dos dados; Guedes SEM foi responsável pela coleta e análise dos dados; Cunha BES foi responsável pela coleta e análise dos dados; Brito CA, responsável pelo desenho, revisão e aprovação final.

Conflito de Interesses: Os autores declaram não haver conflito de interesses

Recebido: 29 de setembro de 2019

Aceito: 19 de novembro de 2020 